\title{
Design of Artificial Neural Networks for Early Detection of Dementia Risk Using Mini-Mental State of Examination (MMSE)
}

Haidar Ali Hamzah ( $\square$ hah057234@gmail.com )

Sriwijaya University

Alyssa Marizki Diazura

Sriwijaya University

Ahmad Emir Alfatah

Sriwijaya University

Theodorus

Sriwijaya University

\section{Research Article}

Keywords: Neural networks, MMSE, dementia

Posted Date: November 17th, 2020

DOI: https://doi.org/10.21203/rs.3.rs-110323/v1

License: (c) (i) This work is licensed under a Creative Commons Attribution 4.0 International License. Read Full License 


\section{Abstract}

Dementia is a brain disorder that interferes with a person's cognitive and behavioral abilities. Unfortunately, late diagnosis is common. Detection of people with suspected dementia in any setting requires a brief and simple test such as MMSE which has become the best-known and the most often used short screening dementia tool. The aim of this study was to determine the diagnostic accuracy of MMSE screening tool based digital system. We used data from the OASIS Longitudinal dataset provided by the University of Washington Center for Alzheimer's Disease Research, Randy Buckner of the Howard Hughes Institute of Medicine at Harvard University, the Neuroinformatics Research Group at the University of Washington School of Medicine, and the Biomedical Informatics Research Network. The dataset was processed through training and testing using an artificial neural network architecture. From this data, a machine learning training process was carried out and obtained diagnostic accuracy of $78 \%$. Thus, The early detection of dementia using MMSE tool based digital system is suitable for use as a dementia risk screening process tool in a clinical setting. Further studies are needed to assess diagnostic accuracy using primary data.

\section{Introduction}

Dementia is a brain disorder that interferes with a person's cognitive and behavioral abilities. People with dementia suffer from behavioral disorders such as apathy, depression, delusions, hallucinations, aggression, irrelevant sexual behavior, and sleep problems. Dementia generally cannot be cured. Before dementia occurs, it is usually marked by Mild Cognitive Impairment $(\mathrm{MCl})$, which is a condition in which individuals experience decreased cognitive abilities that do not interfere with daily activities but are at high risk of developing Alzheimer's disease and dementia (1).Cognitive decline is characterized by impaired learning, memory, language, attention, executive function, and social function (2). The prevalence of $\mathrm{MCl}$ in individuals aged $\geq 65$ years and over is 10$20 \%$, the risk increases with age (3). According to the Alzheimer's Society, $\mathrm{MCl}$ is referred to as a "pre-dementia" condition. A study has shown that $10-15 \%$ of patients suffering from $\mathrm{MCl}$ with memory loss gradually develop dementia each year (4).

The $\mathrm{MCl}$ screening method has become the focus of researchers in recent years. This will help identify patients who are at high risk of dementia so they can be given early treatment and prevention. Unfortunately, late diagnosis is common. This was because most patients or their caregivers did not have access to early detection of dementia and the knowledge to be aware of the symptoms so they were not moved to go to a doctor (5). Detection of people with suspected dementia in any setting requires a brief and simple test of cognitive function or the use of informant questionnaires $(6,7)$. One of the brief cognitive tests is the Mini-Mental State Examination (MMSE) which has become the best-known and the most often used short screening tool for providing an overall measure of cognitive impairment in clinical, research and community settings $(8,9)$.

In this study, we design a dementia risk screening based digital system using MMSE that would allow a doctor to interpret results quickly. The aim of this study was to determine the diagnostic accuracy of MMSE screening tool based digital system.

\section{Methodology}




\subsection{Data}

This study uses secondary data taken from one of Google's open-source sites, Kaggle. This data is published by the Open Access Series of Imaging Studies (OASIS). OASIS is a freely distributed collection of neuroimaging datasets for scientific development that aims to make brain MRI data sets freely available to the scientific community with the hope of facilitating and driving future discoveries in basic and clinical neuroscience. OASIS is provided by the Washington University Alzheimer's Disease Research Center, Dr. Randy Buckner at the Howard Hughes Medical Institute (HMMI) at Harvard University, the Neuroinformatics Research Group (NRG) at Washington University School of Medicine, and the Biomedical Informatics Research Network (BIRN).

There are two OASIS datasets, namely OASIS Cross-sectional and OASIS Longitudinal. This data has been used for data analysis, making anatomical references, and developing algorithms for segmenting brain regions (10). A cross-sectional dataset consisting of a cross-sectional collection of 416 subjects aged 18 to 96 . All subjects were right-handed and included both men and women. 100 subjects over 60 years of age had been clinically diagnosed with very mild to moderate Alzheimer's disease. In addition, a reliability data set was included containing 20 independent subjects who were imaged on subsequent visits within 90 days of their initial session.

The longitudinal dataset consisted of a longitudinal collection of 150 subjects aged 60 to 96 . Each subject was scanned at two or more visits, separated by at least one year for a total of 373 imaging sessions. All subjects were right-handed and included both men and women. 72 subjects were categorized as non-demented during the study. 64 of the included subjects were marked as dementia at their baseline visit and remained so for subsequent scans, including 51 with mild to moderate Alzheimer's disease. Another 14 subjects were categorized as having no dementia at their first visit and then characterized as dementia at the next visit. The dataset also contains additional important information including age, gender, chief complaint, systolic blood pressure, diastolic blood pressure, pulse, and axial head length and head width.

In this study, only the OASIS Longitudinal dataset is used for the training model process using the neural network. Table 1 describes the parameters to be used in the system diagnosis training process.

Table 1. Parameters

\begin{tabular}{|cll|}
\hline No & Parameters & Example \\
\hline 1 & Name & John \\
\hline 2 & Gender & Male/M \\
\hline 3 & Age & 73 \\
\hline 4 & Years of education & 15 \\
\hline 5 & Socioeconomic status & 3 \\
\hline 6 & MMSE & 18 \\
\hline 7 & Category & Moderate \\
\hline
\end{tabular}


The OASIS Longitudinal dataset consists of 373 rows of data of people with dementia and non-dementia which include data such as Subject ID, MRI ID, Group, Visit, MR Delay, M / F, Hand, Age, EDUC, SES. MMSE, CDR, eTIV, nWBV, and ASF. In this study, to create an early detection system for dementia, only a few data were used, such as age, M/F (Gender), EDUC (Years of education), SES (Socioeconomic Status), MMSE (Mini-Mental State Examination), and Group (Converted / Demented / Nondemented) because the data can be obtained easily without the need to go to the hospital so this can make it easier for users to test for early detection of dementia.

In the dataset, 19 rows are identified with missing values in the SES column and 2 rows with missing values in the MMSE column. To solve this problem we have 2 approaches. One is dropping rows with missing values and the other way is replacing missing values with the corresponding values, or known as 'Imputation'. Since the dataset contains only 373 data sets, we assume that imputation will help our model perform better. For the imputation we use mean values because SES and MMSE are discrete variables, so to fill in the null columns we calculate the mean value in that column and fill in the mean value in the null columns.

There are 3 classes in the dataset, namely Nondemented, Demented, and Converted. Converted is a nondemented class which after testing again the result is demented. To make it easier, for converted classes to be replaced with demented so that the dataset only has 2 classes. For the results of the MMSE test, points 24-30 are categorized as Normal, points 19-23 are categorized as Mild, points 10-18 are categorized as Moderate, and points 0-9 are categorized as Severe. The dataset is normalized using Standard Scaler as a feature scaling.

\subsection{Artificial Neural Network (ANN)}

An artificial neural network consists of processing units called neurons. Artificial neurons try to mimic the structure and behavior of natural neurons. A neuron consists of input or dendrite, and one output or synapses via axons. Neurons have a function that determines neuron activation. $x 1$... xn is the input to the neuron. Bias is also added to neurons along with input. Usually, the bias value is initialized to 1 . WO ... Wn is the weight. Weight is the connection to the signal. Product weight and input provide signal strength. A neuron receives many inputs from different sources and has one output. There are various functions used for activation. One of the most commonly used activation functions is the sigmoid function, which is given by

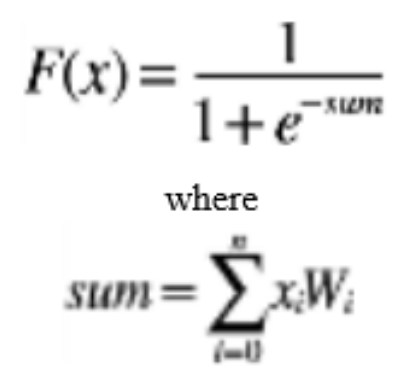

The other functions used are Step Function, Linear Function, Ramp Function, Hyperbolic Tangent Function. The hyperbolic tangent (tanh) function is similar in shape to the sigmoid, but the limit is from -1 to +1 , unlike the sigmoid which is from 0 to 1 . The sum is the sum of the input weights multiplied by the weight between one layer and the next. The activation function used is the sigmoid function, which is a continuous approximation and can be distinguished from the step function (11). Such interconnections of individual neurons form a neural network. 
ANN architecture consists of:

1. input layer: Receives the input value

2. hidden layer (s): A set of neurons between the input and output layers. There can be one or more layers

3. output layer: Usually it has one neuron, and the output is between 0 and 1, that is, greater than 0 and less than

1. But multiple outputs can also be present (12).

Processing capability is stored in the connection strength between units, which is called weights (13). The input power depends on the weight value. Weight values can be positive, negative, or zero. A negative weight means the signal is reduced or inhibited. Zero weight means there is no relationship between the two neurons. Weights are adjusted to get the desired results. There is an algorithm to adjust the weight of the ANN to get the required output. This weight adjustment process is called learning or training (11).

\section{Discussion}

In this research method, we use patient data from the OASIS Longitudinal dataset to analyze the performance of the method. The random dataset is assigned to binary classification and the percentage data is $80 \%$ for training data and $20 \%$ for data testing purposes. Previously, the dataset was processed through training and testing. Neural network architecture uses an architecture with 2 hidden layers and one output layer. From this data, a machine learning training process was carried out and got a diagnostic accuracy of $78 \%$.

\section{Conclusion}

In this study, we used neural network to determine the diagnostic accuracy of MMSE in a digital system. The results indicated that MMSE in a digital system is suitable for use as a dementia risk screening process at a clinical setting. Further study are needed to assess diagnostic accuracy using primary data.

\section{Declarations}

\section{Acknowledgement}

This study was supported by Ministry of Education and Culture under national program Progam Kreativitas Mahasiswa Karsa Cipta. We would like to say thank to Theodorus for assistance during this study.

\section{Conflict of Interest}

There are no conflicts of interest

\section{References}

1. Albert MS, DeKosky ST, Dickson D, Dubois B, Feldman HH, Fox NC, et al. The Diagnosis of Mild Cognitive Impairment due to Alzheimer's Disease: Recommendations from the National Institute on Aging-Alzheimer's Association Workgroups on Diagnostic Guidelines for Alzheimer's Disease. Focus (Madison). 2013;11(1):96106.

2. Psychiatric Association A. The Diagnostic and Statistical Manual of Mental Disorders (DSM-5). 5th ed. Washington: American Psychiatric Publishing; 2013. 
3. Langa KM, Levine DA. The Diagnosis and Management of Mild Cognitive Impairment: A Clinical Review. Expert Rev Neurother. 2009;27(4):590-609.

4. Rodda J. What is mild cognitive impairment (MCI)? Alzheimer's Soc [Internet]. 2015;(August):1-10. Available from:

https://www.alzheimers.org.uk/sites/default/files/pdf/factsheet_what_is_mild_cognitive_impairment_mci.pdf

5. Phillips J, Pond D, Goode SG. Timely Diagnosis of Dementia: Can we do better? A Rep Alzheimer's Aust [Internet]. 2011;(September):23. Available from: www.alz

6. Arevalo-Rodriguez I, Pedraza OL, Rodríguez A, Sánchez E, Gich I, Solà I, et al. Alzheimer's disease dementia guidelines for diagnostic testing: A systematic review. Am J Alzheimers Dis Other Demen. 2013;28(2):111-9.

7. Moyer VA. Screening for Cognitive Impairment in Older Adults: U.S. Preventive Services Task Force Recommendation Statement. Ann Intern Med. 2003;157(5):1-10.

8. Nieuwenhuis-Mark RE. The death knoll for the MMSE: Has it outlived its purpose? J Geriatr Psychiatry Neurol. 2010;23(3):151-7.

9. Folstein MM, Folstein SE. Mini-Mental State A Practical Method for Grading The Cognitive State of Patients for The Clinician. J Psychiatr Res. 1974;12:189-98.

10. Marcus DS, Fotenos AF, Csernansky JG, Morris JC, Buckner RL. Open access series of imaging studies: Longitudinal MRI data in nondemented and demented older adults. J Cogn Neurosci. 2010;22(12):2677-84.

11. Gershenson C. Artificial neural networks for Beginners. ICES J Mar Sci. 2009;66(6):1119-29.

12. Shukla M, Abdelrahman M. Artificial Neural Networks based steady state security analysis of power systems. Proc Annu Southeast Symp Syst Theory. 2004;36:266-9.

13. Kuldeep S, Anitha GS. Neural Network Approach for Processing Substation Alarms. Int Journals Power Electron Control Convert. 2017;1(1):21-8.

\section{Figures}






Figure 1

Model of artificial neuron 


\section{Input layer}

Hidden layer

Output layer

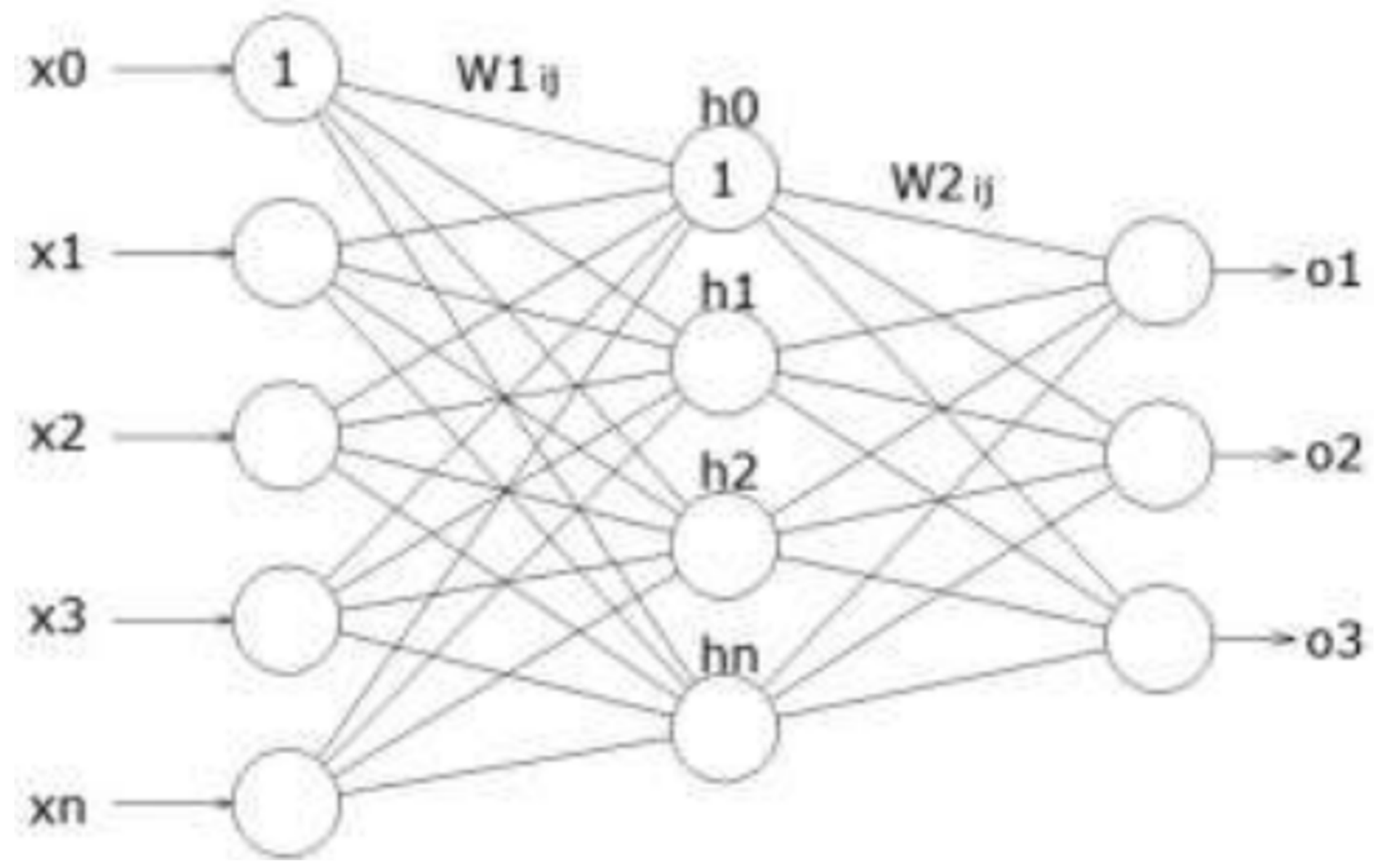

Figure 2

Neural network architecture 